\title{
Determinants of Gastroesophageal Reflux Disease (GERD) severity and Utilization of Proton Pump Inhibitors among GERD Patients: A Cross-Sectional Study
}

\author{
Jinin S. Mathew ${ }^{1}$, M. R. V. Nandini ${ }^{1}$, G. R. Saraswathy ${ }^{2}$, Viswam Subeesh ${ }^{2}$, Eswaran Maheswari ${ }^{2}$ \\ ${ }^{1}$ Dept. of Pharmacy Practice, Faculty of Pharmacy, M.S. Ramaiah University of Applied sciences, Bangalore-560054, Karnataka. \\ ${ }^{2}$ Dept. of Pharmacy Practice, Faculty of Pharmacy, M.S. Ramaiah University of Applied sciences, Bangalore-560054, Karnataka.
}

\author{
ARTICLE INFO \\ Article history: \\ Received on: 28/08/2017 \\ Accepted on: 18/09/2017 \\ Available online: 30/11/2017 \\ Key words: \\ Proton pump inhibitors \\ (PPIs), Gastro-esophageal \\ reflux disease (GERD), \\ prescription pattern, non- \\ erosive reflux disease \\ (NERD), erosive esophagitis \\ (EE).
}

\begin{abstract}
Background: Proton Pump Inhibitors (PPIs) are the first line agents in the treatment of gastro-esophageal reflux disease (GERD). The efficiency and safety profile of PPIs have driven to its over-utilization and the protracted recipients are targets to its long-term adverse such as chronic kidney disease (CKD), Clostridium difficile infection (CDI) and community-acquired pneumonia (CAP) etc. Therefore, the study was intended to explore the PPI utilization patterns in GERD patients in an Indian tertiary-care hospital.

Materials and Methods: A cross-sectional study was carried out at the Department of Gastroenterology, in a private tertiary care hospital, Bangalore, for a period of six months. The objective of the study was to identify the risk factors of GERD and prescribing trends of proton pump inhibitors in GERD patients.

Results: Among the 139 patients diagnosed with GERD, 66\% were males. GERD was found to be more prevalent in the age group of 25-44 years. The GERD patients reported a relationship between the severity of disease and the following risk factors, namely, Smoking, OR: 0.446, alcoholism, OR: 1.26, BMI>24.9, OR:1.13 and mixed diet, OR:0.486. Rabeprazole (36.4\%) was the commonly prescribed PPI. PPI combination therapy was prescribed in $56.11 \%$ of patients, followed by single therapy in $39.5 \%$ patients.
\end{abstract}

Conclusion: Creating awareness among the clinicians and patients on appropriate use of PPIs can reduce the additional economic burden and improve the health-related quality of life. Focusing on risk factors to identify preventive measures will help in decreasing the incident rate of GERD.

\section{INTRODUCTION}

Proton pump inhibitors (PPIs) are widely prescribed classes of medications, used in the treatment of gastrointestinal (GI) disorders. They act by inhibiting the $\mathrm{H}+\mathrm{K}+$-adenosine triphosphatase (ATPase), or proton pump, located in the highly acidic lumen of parietal cells (Blume et al., 2006). This highly acidic environment allows the conversion of PPI to its protonated active state which then irreversibly inhibits the activity of the

* Corresponding Author

Subeesh K Viswam, Assistant Professor, Dept. of Pharmacy Practice, Faculty of Pharmacy, M.S. Ramaiah University of Applied Sciences, Bangalore-560054, Karnataka, India. Phone no: +91-9448471346, +91-9895843219; Email: subeeshkviswam @ gmail.com proton pump, leading to increase of gastric $\mathrm{pH}$. This class of medications has transformed the way in which clinicians treat the acid-related disorders of the GI tract. They have become the first line treatment of gastro esophageal reflux disease (GERD), peptic ulcer disease (PUD), and helicobacter eradication, the most commonly experienced conditions that results in upper GI problems (Chiba et al., 2013). The use of these drugs has increased by $456 \%$ since the 1990's (Eid et al., 2010). As a result, these drugs have become one of the most frequently prescribed medications in the world. Several clinical trials have shown that PPIs are both well tolerated and safe when used acceptably (Chiba et al., 2013; Nousheen et al., 2014; Singh et al., 2011; Airee et al., 2016). However, the overuse of PPI's followed by its misuse increases the plausibility of adverse effects. 
Protracted usage of PPIs can lead to its long term adverse effects such as chronic kidney disease (CKD), Clostridium difficile infection (CDI), community-acquired pneumonia (CAP), osteoporosis etc (Gikas et al., 2014, Ito et al., 2010, Heidelbaugh et al., 2012). Since, long-term treatment is required for most of the gastric-acid related disorders, in patients who are receiving other medications in conjunction with a PPI, the risk for clinically significant adverse drug interactions are probable. Several studies have reported that PPIs are being overused in hospital as well as in the ambulatory care settings and also the appropriateness of PPI prescriptions in some hospitals is as low as $19 \%$ (Naunton et al., 2000). The incidence of inappropriate usage of PPIs varies from $40-70 \%$ in different studies. Initiating the drug therapy inappropriately and its longterm use will result in the increased economic burden for the patient. Awareness should be created among the clinicians so that PPIs are prescribed appropriately which helps in improved patient care at lower costs (Pasina et al., 2010; Nousheen et al., 2014).

The objective of the study was to explore the prescribing pattern of PPIs in GERD in a tertiary care hospital, Bangalore, as there was a presumptive evidence that the PPIs are not being appropriately used (Joel et al., 2010). The study was also focused on identifying the determinants of GERD severity.

\section{MATERIALS AND METHODS}

\section{Study design and setting}

This cross-sectional study was conducted in a private multi-specialty 750 bed tertiary care hospital in Bangalore, for a period of six months from November 2015-April 2016. This study was approved by the Institutional Ethics Committee.

The study enrolled 139 GERD patients visiting the outpatient department of gastroenterology, with the endoscopic evidences. Patients of either gender (aged > 18 years) attending to gastroenterologists OPD were eligible for inclusion, if they presented with typical symptoms of GERD (heartburn, regurgitation) with the presence or absence of extra esophageal symptoms more than twice a week for at least 2 months. Patients who were not willing to sign the consent, patients in whom confirmatory diagnosis was not obtained, patients with history of unsuccessful PPI therapy and patients with indication for nonpharmacological therapy (Hiatal hernia) were excluded from the study. The patient's medication charts, clinical history, laboratory data and other relevant data were reviewed and necessary data were collected according to the objective of the study in a suitably designed data collection form. The pooled data were then analyzed to find the prescribing pattern of PPIs in GERD.

\section{Statistical analysis}

Statistical analysis of the obtained data was performed using SPSS. The correlation between the risk factors and GERD studied using Pearson's $\chi 2$ test. The threshold for significance was set at $p<0.05$. The strength of relation between each variable and risk of GERD was evaluated by bivariate logistic regression model. Variables with odds ratio $(\mathrm{OR})>1$ and $\mathrm{p}<0.05$ were considered significant risk factors for GERD.

\section{RESULTS}

Among the 139 patients, 66\% ( $n=92)$ were males. The male to female ratio in occurrence of GERD was found to be $2: 1$. The mean age of the patients with GERD was found to be $41.97 \pm 14.10$ years. There were $54 \%$ of patients who belonged to the age group 25-44 years among which $70.6 \%(n=53)$ were male and $29.4 \%(n=22)$ female. In total study subjects, $64.7 \%(n=90)$ cases were NERD and $35.3 \%(n=49)$ cases were EE. The details are specified in Figure 1.

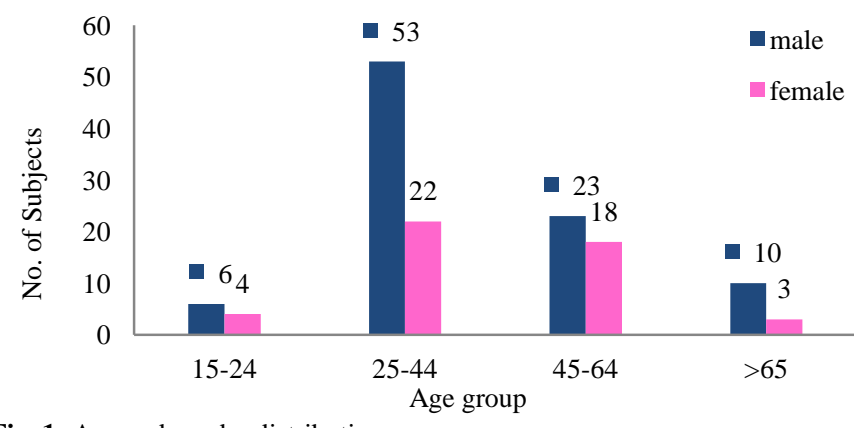

Fig. 1: Age and gender distribution

The GERD patients reported a relationship between the severity of disease and risk factors (Smoking $\mathrm{P}=0.047$, Alcoholism $\mathrm{P}=0.017$, BMI $\mathrm{P}=0.027$ and Diet $\mathrm{P}=0.048$ ). The bivariate logistic regression analysis showed following risk factors: smoking $(\mathrm{OR}=$ 0.446, 95\% CI: 0.199-0.998, $\mathrm{P}=0.309)$, Alcohol consumption (OR $=1.26,95 \%$ CI: $0.693-2.46, \mathrm{P}=0.047), \mathrm{BMI}>24.9 \mathrm{~kg} / \mathrm{m}^{2}(\mathrm{OR}=$ 1.13, 95\% CI: $0.564-2.23, \mathrm{P}=0.046)$ and mixed diet $(\mathrm{OR}=0.486$, 95\% CI: $0.237-0.998, \mathrm{P}=0.036)$. The details are specified in Table 1.

Table 1: Results of logistic regression analysis of risk factors for GERD.

\begin{tabular}{|c|c|c|c|c|c|c|}
\hline \multirow{2}{*}{ Covariate } & \multirow{2}{*}{ Pearson correlation } & \multirow{2}{*}{ P value } & \multirow{2}{*}{ Odds ratio } & \multicolumn{2}{|c|}{ 95\% confidence interval } & \multirow{2}{*}{ P value } \\
\hline & & & & Lower & Upper & \\
\hline Smoking & 0.169 & 0.047 & 0.446 & 0.199 & 0.998 & 0.309 \\
\hline Alcoholism & 0.203 & 0.017 & 1.26 & 0.693 & 2.46 & 0.047 \\
\hline BMI > 24.9 & 0.188 & 0.027 & 1.13 & 0.564 & 2.23 & 0.046 \\
\hline Mixed diet & 0.168 & 0.048 & 0.486 & 0.237 & 0.998 & 0.036 \\
\hline
\end{tabular}




\section{Utilization pattern of PPI}

A total of 170 PPIs were utilized. The most commonly prescribed PPI was rabeprazole $36.4 \%$, followed by pantoprazole $33.5 \%$ and esomeprazole $25.8 \%$.

Combination therapy was prescribed in $56.2 \%(n=78)$ of patients, followed by single therapy in $39.5 \%(n=55)$ patients and $4.3 \%(n=6)$ of patients were treated with other agents like $\mathrm{H}_{2}$ RAS $\left(\mathrm{H}_{2}\right.$ receptor antagonists) and antacids. The details are specified in Table 2.

Table 2: Pattern of therapy in study population.

\begin{tabular}{ccc}
\hline Therapy & Number of Patients & Percentage (\%) \\
\hline Single & 55 & 39.5 \\
Combination therapy & 78 & 56.2 \\
Others & 6 & 4.3 \\
Total & 139 & 100 \\
\hline
\end{tabular}

\section{PPI utilization in NERD}

Among NERD patients $(n=90)$, a total of 107 PPIs were used. $65.5 \%$ of the patients were male $(n=59)$. The most commonly prescribed PPI was rabeprazole $36.44 \%$ followed by pantoprazole $35.5 \%$ and esomeprazole $23.6 \%$. Combination therapy (PPI + prokinetics) was found to be prescribed in $54.4 \%($ $\mathrm{n}=49)$ of the patients, $40 \%(\mathrm{n}=36)$ with single therapy and $5.6 \%($ $\mathrm{n}=5$ ) were prescribed with other drugs like $\mathrm{H}_{2} \mathrm{RAS}$ and antacids. Single therapy was given to $40 \%(n=36)$ patients, $44.44 \%(n=18)$ of the patients received pantoprazole, $25 \%(n=10)$ rabeprazole and $22.2 \%(n=8)$ esomeprazole. The details are specified in Table 3.

\section{PPI utilization in EE}

In EE $(n=49)$ patients, a total of 62 PPIs were used. The most commonly prescribed PPI was rabeprazole $36.5 \%$ followed by pantoprazole $30.1 \%$ and esomeprazole $30.24 \%$. Combination therapy (PPI + prokinetics) was given to $59.1 \%$ of the patients,
$38.7 \%$ were treated with single therapy and $2.2 \%$ were not prescribed any PPIs. Among the patients prescribed with single therapy, $52.8 \%$ were given with esomeprazole, $26.3 \%$ with pantoprazole and $15.7 \%$ with rabeprazole. The details are specified in Table 3.

\section{Combination therapy in NERD}

Among the patients prescribed with PPI combination therapy $(n=49), 89.75 \%$ were given with PPI + prokinetics combination and $10.3 \%$ given with PPI + antibiotic combination. The most commonly used PPI in the PPI+ prokinetics combination was rabeprazole i.e., $54.34 \%$ and in PPI + antibiotic combination esomeprazole was commonly used. The details are specified in Table 4.

\section{Combination therapy in EE}

All the patients in whom PPI combination therapy was advised, received PPI + prokinetics combination $(n=30)$. Rabeprazole + prokinetic combination was found to mostly prescribed, $65.5 \%$ followed by pantoprazole + prokinetics combination $20.6 \%$, esomeprazole + prokinetics $10.3 \%$ and omeprazole +prokinetics $3.44 \%$. The details are specified in Table 4

\section{Conversion therapy in NERD}

Conversion therapy was practiced among $31.1 \%(n=28)$ of the total NERD patients $(\mathrm{n}=90)$. The most frequent conversion was PPI to PPI + prokinetics $25 \%(\mathrm{n}=7)$ and PPI + prokinetics to PPI + prokinetics $25 \%(n=7)$. PPI to another PPI conversion was observed in 6 patients, 5 underwent PPI to PPI + antibiotic conversion and conversion from PPI + antibiotic to PPI with prokinetics was seen in 3 patients. The details are specified in Table 5 .

Table 3: PPIs utilization pattern in GERD patients.

\begin{tabular}{|c|c|c|c|c|c|c|}
\hline \multirow[t]{2}{*}{ Type of GERD } & \multicolumn{5}{|c|}{ Frequency of PPIs prescribed } & \multirow[t]{2}{*}{ Total number of PPIs } \\
\hline & Pantoprazole & Rabeprazole & Omeprazole & Esomeprazole & Ilaprazole & \\
\hline NERD & $38(35.5 \%)$ & $39(36.44 \%)$ & $5(4.6 \%)$ & $25(23.6 \%)$ & 0 & $107(62.9 \%)$ \\
\hline EE & $19(30.1 \%)$ & $23(36.5 \%)$ & $1(1.58 \%)$ & $19(30.24 \%)$ & $1(1.58 \%)$ & $63(37 \%)$ \\
\hline
\end{tabular}

Table 4: Pattern of combination therapy in GERD.

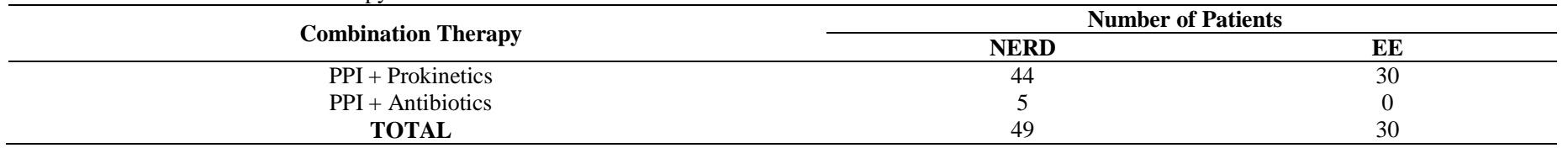

Table 5: Pattern of conversion therapy in GERD.

\begin{tabular}{|c|c|c|}
\hline \multirow[t]{2}{*}{ Pattern of Conversion } & \multicolumn{2}{|c|}{ Number of Patients } \\
\hline & $\mathbf{E E}$ & NERD \\
\hline PPI to another PPI & 1 & 6 \\
\hline PPI to PPI + Prokinetics & 12 & 7 \\
\hline PPI to PPI + Antibiotics & 0 & 5 \\
\hline PPI + Prokinetics to PPI + Prokinetics & 2 & 7 \\
\hline PPI + antibiotic to PPI with prokinetics & 0 & 3 \\
\hline Total & 15 & 28 \\
\hline
\end{tabular}




\section{Conversion therapy in EE}

In a total of $49 \mathrm{EE}$ patients, $30.6 \%(n=15)$ of patients underwent conversion therapy. The most frequent conversion were PPI to PPI + prokinetics $80 \%$ and PPI + prokinetics to another PPI + prokinetics $13.3 \%$. Only one patient underwent PPI to another PPI conversion. PPI to PPI + prokinetics conversion therapy was given to $20.4 \%$ patients. The details are specified in Table 5 .

\section{Duration of therapy in NERD}

The mean duration of therapy was found to be $3.94 \pm 2.43$ weeks and $90.5 \%$ of patients prescribed with $<8$ weeks therapy.

\section{Duration of therapy in EE}

The mean duration of therapy was found to be $4.75 \pm$ 2.62 weeks $91.5 \%$ of the patients prescribed with $<8$ weeks of therapy.

\section{DISCUSSION}

Proton pump inhibitors (PPIs) are one of the most commonly prescribed classes of medications in the hospital settings and are considered as a major advance in the treatment of GERD (Singh VP, Prabhu K et al., 2011). PPIs in general cause fewer adverse effects and have a better tolerability which has probably led to their inappropriate use (Airee et al., 2016). This study address the utilization of PPI among GERD patients.

The study enrolled 139 patients diagnosed with GERD by the gastroenterologist, among which two third were men. The result obtained from this study was in accordance with the results of the study conducted by Kiyotaka et al., in 2016 who had reported that GERD is more prevalent in males due to their body mass, lifestyle (Kiyotaka et al., 2016).The studies conducted worldwide on various populations showed that risk factors for GERD include age, excessive body mass, lifestyle factors (such as smoking, physical activity), and diet (Nilsson et al., 2004, Zheng et al., 2007, Lee et al., 2011). Concordantly our study showed a significant relationship between the severity of GERD and risk factors like smoking, alcoholism, abnormal BMI $\left(>24.9 \mathrm{~kg} / \mathrm{m}^{2}\right)$ and mixed diet, however alcoholism and abnormal BMI $(>24.9$ $\mathrm{kg} / \mathrm{m}^{2}$ ) were the risk factors for GERD in our study.

More than half of the study population belonged to the age group of 25-44 years, which is in accordance with the study by Moshkowitz et al in the year 2011 also showed that majority of the study subjects belonged to the similar age group (Moshkowitz et al., 2011).

Study revealed that $56.11 \%$ of the patients were treated with combination therapy followed by $39.5 \%$ patients with monotherapy therapy and $4.3 \%$ of patients with agents other than PPIs. Rabeprazole and pantoprazole were found to be utilized more compared to the other drugs in the same category. The study site was a tertiary care hospital, where patients with poor economic background accounts for a good proportion. By considering the fact that rabeprazole is the cheaper option among the category, it was utilized more. In the light of literatures, it's known that esomeprazole is more effective in relieving reflux symptoms of GERD when compared to lansoprazole, omeprazole, pantoprazole and rabeprazole (Robert and Lowe, 2006). But a pharmaco-economic evaluation is required to compare the cost effectiveness among PPIs to support the aforementioned claim.

Prokinetic drugs like domperidone or levosulpiride were prescribed with PPI as combinations. The study carried out by Ndraha in the year 2011 divulged that combination of omeprazole with domperidone in GERD patients was more superior compared to omeprazole monotherapy (Ndraha, 2011). A study conducted by Khajuria et al., also disclosed that combination therapy of pantoprazole and prokinetic (mosapride) was superior to pantoprazole alone (Khajuria et al., 2016). The former research support the fact that PPI combination with prokinetics have a beneficiary effect in comparison with monotherapy. More randomized control trials are required to support this claim.

The antibiotics use can be rational only in case of patients having other infections as mentioned in the research paper by Yearsley KA et al., in 2006 (Yearsley et al., 2006). However the use of antibiotics in the current study population cannot be justified with the aforesaid claim. In our study, conversion or switching of $\mathrm{PPI}_{1}$ to $\mathrm{PPI}_{2}, \mathrm{PPI}$ to $\mathrm{PPI}+$ prokinetics, $\mathrm{PPI}+$ prokinetics to another PPI+prokinetics combinations were noticed. These switching of drugs can be mainly due to decreased healing rates, side effects, resistance or due to increased cost of therapy. The most common conversion was PPI+prokinetics to another PPI+prokinetics.

The mean duration of therapy was 3-5weeks among NERD cases and 4-6 weeks among EE cases. Less than 8 weeks of therapy was prescribed for majority the study population (91\% NERD and $91.6 \% \mathrm{EE})$. This result was in concordance with the study conducted by Dean et al., in 2004 where the duration of therapy was reported to be 4 weeks (Dean et al., 2004). According to ACG guideline, the duration of therapy should be 8 weeks (Katz et al., 2013). It is justifiable to deviate from the ACG guideline as it was developed and practiced in a different ethnic population. However, the symptom relief and remission rate should exhibit a promising result in population in whom there was deviation from the standard guideline.

Short duration of time as well as the small sample size accounted to be the limitations of this study. In addition, the clinical outcomes need to be studied even though the protocol followed by the hospital in the treatment of GERD is competent.

\section{CONCLUSION}

Rabeprazole was the most commonly prescribed PPI.The pattern of prescription of PPIs and duration of therapy were found to vary to a higher degree in GERD patients. Awareness should be created among the health care professionals and patients as the inappropriate use of PPIs can result in poor health related quality of life. Focusing on risk factors to identify preventive measures will help in decreasing the incident rate of GERD. 


\section{ACKNOWLEDGEMENT}

We sincerely thank our Principal, Dr. V. Madhavan for giving us an opportunity and providing us the resources and support during our project work.

\section{Financial support and sponsorship: Nil.}

Conflict of Interests: There are no conflicts of interest.

\section{REFERENCES}

A kiyotaka, L Katsunori, S Tooru. Gender difference in gastroesophageal reflux diseases. World J Gastroenterol, 2016; 22:1800-1810.

Airee RS, Rawal A, John NN, Binu KM. Drug use evaluation of proton pump inhibitors in a private tertiary care teaching hospital. World $\mathrm{J}$ Pharm Pharm Sci, 2016; 5:923.

Blume H, Donath F, Warnke A, Schug B. Pharmacokinetic drug interaction profiles of proton pump inhibitors. Drug Safety, 2006; 29:76980 .

Chiba T, Malfertheiner P, Satoh H. Proton Pump Inhibitors: A Balanced View. Karger, 2013; 32:59-67.

Dean BB, Gano AD, Knight, Ofman JJ, Fass R. Effectiveness of proton pump inhibitors in nonerosive reflux disease. Clin Gastroenterol Hepatol, 2004; 2:656-64.

Eid A, Boueiz S, Paranji C, Mativo R, Landis M, Abougergi. Patterns and predictors of proton pump inhibitor overuse among academic and non-academic hospitalists. Internal Med, 2010; 49:2561-8.

Gikas K, Triantafillidis JK. The role of primary care physicians in early diagnosis and treatment of chronic gastrointestinal diseases. Int J Gen Med, 2014; 13(7):159-73.

Heidelbaugh JJ, Kim AH, Chang R, Walker PC. Overutilization of proton-pump inhibitors: what the clinician needs to know. Therap Adv Gastroenterol, 2012; 5(4):219-32.

Ito $\mathrm{T}$, Jensen RT. Association of long-term proton pump inhibitor therapy with bone fractures and effects on absorption of Calcium, vitamin B12, iron, and magnesium. Curr Gastroenterol Rep, 2010; 2(6):448-57.

Joel J, Heidelbaugh, Kathleen L, Goldberg, John MI. Magnitude and economic effect of overuse of antisecretory therapy in the ambulatory care setting. Curr Clin Pharmacol, 2010; 5:288-97.

Katz PO, Gerson LB, Vela MF. Guidelines for the diagnosis and management of gastroesophageal reflux disease. Am J Gastroenterol, $2013 ; 3: 308-28$

Khajuria DK, Vinod K, Razavi M, Karimian H, Pradeep S. Gastroesophageal reflux disease: A Review of Superior Effects of Proton Pump Inhibitors and Prokinetic agents as a Combination Therapy versus Monotherapy. Pharmacologyonline, 2016; 3:701-5.
Lee SJ, Jung MK, Kim SK, Jang BI, Lee SH, Kim KO, Kim ES, Cho KB, Park KS, Kim EY, Jung JT. Clinical characteristics of gastroesophageal reflux disease with esophageal injury in Korean: focusing on risk factors. Korean J Gastroenterol, 2011; 57:281-7.

Moshkowitz M, Horowitz N, Halpern Z, Santo E. Gastroesophageal reflux disease symptoms: Prevalence, sociodemographics and treatment patterns in the adult Israeli population. World J Gastroenterol, 2011; 17:1332-35.

Naunton M, Peterson G, Bleasel M. Overuse of proton pump inhibitors. J Clin Pharm Ther, 2000; 25:333-8.

Ndraha S. Combination of PPI with a Prokinetic Drug in Gastroesophageal Reflux Disease. Acta Media Indonesia-Indonesia Journal of Internal Medicine, 2011;43:4

Nilsson M, Johnsen R, Ye W, Hveem K, Lagergren J. Lifestyle related risk factors in the aetiology of gastro-oesophageal reflux. Gut, 2004; 53:1730-5.

Nousheen, Tadvi NA and Shareef SM. Use of proton pump inhibitors in general practice: Is it rationale. Int J Med Res Health Sci, 2014; 3:37-42.

Pasina L, Nobili A, Tettamanti M, Salerno F, Corrao S, Marengoni A et al. Prevalence and appropriateness of drug prescriptions for peptic ulcer and gastroesophageal reflux disease in a cohort of hospitalized elderly. Am J Managed Care, 2010; 16:228-34.

Robert C, Lowe MD. Medical management of gastroesophageal reflux disease. GI Motility online, 2006; 54:1038.

Singh VP, Prabhu K, Ponnudurai K, Singh PK. Prescribing pattern of acid suppressants in modern clinical practice - An analysis. Der Pharmacia Sinica, 2011; 2:67-73.

Yearsley KA, Gilby LJ, Ramadas AV, Kubiak EM, Fone DL, Allison MC. Proton pump inhibitor therapy is a risk factor for Clostridium difficile-associated diarrhoea. Ailment Pharmacol Ther, 2006; 10:613-9.

Z Zheng, H Nordenstedt, NL Pedersen, W Ye, J Lagergren. Lifestyle factors and risk for symptomatic gastroesophageal reflux in monozygotic twins. Gastroenterology, 2007; 132:87-95.

\section{How to cite this article:}

Mathew JS, Nandini MRV, Saraswathy GR, Subeesh V, Maheswari E. Determinants of Gastroesophageal Reflux Disease (GERD) severity and Utilization of Proton Pump Inhibitors Among GERD Patients: A Cross-Sectional Study. J App Pharm Sci, 2017; 7 (11): 172-176. 